\title{
El Real Colegio de Cirugía de Nueva España, en la Ciudad de México: a 250 años de su fundación
}

\author{
The Royal College of Surgery of New Spain, in Mexico City: 250 years ago of its \\ foundation
}

\author{
Ulises Rodríguez-Wong ${ }^{\text {* }}$ y Ulises Rodríguez-Medina ${ }^{2}$ \\ ${ }^{1}$ Academia Mexicana de Cirugía; ${ }^{2}$ Facultad Mexicana de Medicina, Universidad La Salle. Ciudad de México, México
}

\begin{abstract}
Resumen
El Real Colegio de Cirugía de Nueva España, en la Ciudad de México, fue establecido por decreto del Rey Carlos III, en España, el día 17 de marzo de 1768. Dos cirujanos provenientes de los Reales Colegios de Cirugía de Cádiz y Barcelona fueron enviados al nuevo continente para iniciar las actividades de este colegio. Los cirujanos españoles peninsulares trajeron consigo los conocimientos más recientes de la cirugía europea, así como métodos más racionales en el tratamiento quirúrgico de algunas enfermedades. El Real Colegio de Cirugía permitió normar y profesionalizar la práctica de la cirugía en la Nueva España.
\end{abstract}

PALABRAS CLAVE: Cirugía en México. Colegio de Cirugía. Real Colegio de Cirugía.

\begin{abstract}
The Royal College of Surgery of New Spain, in Mexico City, was established by decree of King Carlos III, in Spain, on March 17, 1768. Two surgeons from the Royal Colleges of Surgery of Cádiz and Barcelona were sent to the new continent, to start the activities of this school. The peninsular Spanish surgeons brought with them the most recent knowledge of European surgery, as well as more rational methods in the surgical treatment of some diseases. The Royal College of Surgery allowed to regulate and professionalize the practice of Surgery in New Spain.
\end{abstract}

KEY WORDS: Surgery in Mexico. College of Surgery. Royal College of Surgery.

\section{Introducción}

El 17 de marzo de 1768, en Aranjuez, España, Carlos III decretó el establecimiento del Real Colegio de Cirugía de Nueva España, en la Ciudad de México", con la finalidad de realizar actividades semejantes a las de los Reales Colegios de Cirugía ubicados en Cádiz y en Barcelona, en la región peninsular. Este decreto fue refrendado por Cédula Real el 20 de mayo del mismo año. Esto culminó el esfuerzo realizado por Don Antonio Arroyo, quien había presentado la propuesta varios años atrás. Es por ello que el Instituto Nacional de Estadística y Geografía de México reconoce como fecha de la fundación de este Colegio el año de $1768^{2}$.

El decretó permitía la impartición de la cátedra de Anatomía Práctica en el Hospital Real de Naturales en la Ciudad de México, pero al constituirse el Colegio

\author{
Correspondencia: \\ *Ulises Rodríguez-Wong \\ Tepic, 113, interior 611 \\ Col. Roma Sur \\ C.P. 06760, Ciudad de México, México \\ E-mail: ulisesromed@prodigy.net.mx
}

Fecha de recepción: 29-05-2018

Fecha de aceptación: 15-12-2018

DOI: $10.24875 / C I R U .18000490$
Cir Cir. 2019;87:595-599

Contents available at PubMed www.cirugiaycirujanos.com 
se agregaron las cátedras de Fisiología y Cirugía. Estas cátedras surgieron de manera paralela a las cátedras que ya impartía la Real y Pontificia Universidad de México a los estudiantes de Medicina; sin embargo, el Real Colegio de Cirugía de Nueva España en la Ciudad de México fue la única institución creada por decreto real específicamente para la enseñanza de la cirugía. Fue, quizás, uno de los primeros centros de enseñanza de este tipo en el continente americano.

El Real Colegio de Cirugía se instauró frente al tradicionalismo de la Facultad de Medicina de la Universidad de México y la vanguardia de la Universidad de Guadalajara ${ }^{3}$. Aunque el Real Colegio nunca pudo superar la tradición y el peso académico de la Universidad, ni tampoco pudo igualar la autoridad que ejercía en la Nueva España el Tribunal del Protomedicato, gracias a él se logró la profesionalización de la práctica de la cirugía en la Nueva España, sentando las bases para que más adelante, en el México independiente, se fusionaran la enseñanza de la Medicina y la de la Cirugía.

\section{Primeros nombramientos}

Dos cirujanos peninsulares fueron designados para iniciar las actividades de este nuevo Colegio4: Andrés Montaner y Virgili, como Director y Primer Catedrático, y Manuel Antonio Moreno Rodríguez, como Disector.

Andrés Montaner y Virgili era sobrino del brillante cirujano Pedro Virgili, y había sido destacado alumno del Real Colegio de Cirugía de Cádiz, donde se graduó como Cirujano Primero. Manuel Antonio Moreno Rodríguez era hijo del cirujano del Regimiento de Infantería de Granada Don Bartolomé Moreno, quien había fallecido en 1773 en la Nueva España. Moreno también realizó sus estudios de cirugía en el Real Colegio de Cirugía de Cádiz, donde con toda seguridad fue condiscípulo de Montaner, ya que fue este quien lo propuso como Disector del Real Colegio de Cirugía de la Nueva España, en la Ciudad de México.

En septiembre de 1768, Montaner y Moreno se embarcaron en el puerto de Cádiz hacia la Nueva España en el navío El Dragón. Con los 5000 reales que les asignaron a Montaner y Moreno se compraron dos cajas de instrumental y los libros de Cirugía que fueron autorizados por el Santo Oficio; entre ellos se encontraban Compendio de la Medicina Práctica de Alen, Tratado de Hernias de Arnaud, Obras de Cirugía de Ambrosio Paré, Exposición Anatómica de Winslow,
Operaciones de Cirugía de Velasco y Villaverde, y Phisiologia de Haller, entre otros ${ }^{5}$.

A su llegada a la Ciudad de México, Montaner y Moreno se encontraron con algunas dificultades, ya que no había instalaciones adecuadas en el Hospital Real de Naturales y, por otra parte, el entonces Cirujano Primero Domingo Russi se negó a entregarles las salas de cirugía del hospital, lo que ponía de manifiesto la rivalidad que existía entre los españoles peninsulares y los españoles nacidos en la Nueva España, los criollos. En realidad, Russi tenía razón en cuanto a que Montaner y Moreno únicamente tenían nombramiento de catedráticos, pero no como cirujanos del Hospital de Real de Naturales, y además Russi tenía un nombramiento como cirujano interino. El conflicto tuvo que ser finalmente resuelto por el rey, quien el 17 de abril de 1771 instruyó al virrey de Croix para que diera posesión de sus cargos a Montaner y Moreno.

\section{Inicio de las actividades}

Montaner solicitó la intervención del virrey Carlos Francisco de Croix para construir un segundo anfiteatro anatómico. Según Rómulo Velasco Ceballos en su obra La Cirugía Mexicana en el siglo xviii, el anfiteatro debería medir 12 varas por lado (aproximadamente 10 metros), contar con estantes y vitrina, una mesa de disección ovalada de mármol, y gradas y sillones para los asistentes distinguidos, siendo el del Director más ancho y de respaldo más alto ${ }^{6}$.

$\mathrm{Ni}$ el Hospital Real de Naturales ni el Real Colegio de Cirugía de la Nueva España contaban con los recursos económicos para llevar a cabo este proyecto, por lo que tuvo que habilitarse el anfiteatro ya existente en el nosocomio. De esta manera, el 3 de febrero de 1770, a las 10 de la mañana, el cirujano Andrés Montaner y Virgili presentó oficialmente la primera demostración de anatomía ${ }^{7}$. El 10 de abril de 1770, el virrey marqués de Croix anunció la existencia del Colegio como único centro de instrucción quirúrgica, de tal manera que ningún cirujano podría presentar examen ante el Tribunal del Protomedicato si no contaba con la certificación de haber realizado los cuatro cursos de cirugía del Colegio.

Aquellos cirujanos que tuvieran cubiertos parcialmente los años de entrenamiento debían completar su instrucción en el Real Colegio de Cirujanos, en tanto que los que ya tuvieran completo su entrenamiento debían certificarse mediante un examen de aptitud para finalmente poder presentar su examen ante el 
Protomedicato. De esta manera se profesionalizó el ejercicio de la Cirugía en la Nueva España.

Gracias al establecimiento del Real Colegio de Cirugía, los cirujanos novohispanos accedieron a una enseñanza institucional que intentaba incorporar al ejercicio quirúrgico los modernos conocimientos teóricos y prácticos, cuya adquisición derivó en el cuestionamiento de la tradicional organización gremial de las profesiones de la salud. El obtener nuevos conocimientos implicó un cambio de tipo curricular en la formación de los cirujanos y trajo consigo la generación de una nueva concepción de la Medicina como profesión y como práctica ${ }^{8}$.

Durante este periodo llegan a la Nueva España infinidad de obras de medicina, cirugía y obstetricia, que abren nuevos horizontes al estudio, acaban con las añosas tendencias hipocráticas, borran las normas avicénicas y muestran a los estudiantes senderos racionales $^{9}$. Los profesores del Real Colegio de Cirugía basaron sus sistemas de enseñanza en los estudios de anatomía, la práctica de disecciones en cadáveres y el estudio clínico completo de los pacientes ${ }^{10}$.

En el año de 1781, el duque de Terranova y Montenegro informó al Consejo de Indias de que el Hospital de Jesús fungía como centro de formación de practicantes de Cirugía. El Consejo de Indias dictaminó que estos practicantes deberían de asistir a los cursos del Real Colegio de Cirugía, permitiéndoles seguir desempeñándose como practicantes en dicho hospital, haciendo extensiva esta disposición a otros hospitales como el de San Juan de Dios y el de San Andrés.

Las materias que se impartían en el Real Colegio de Cirugía eran la de Anatomía Práctica, la de Operaciones de Cirugía, la de Fisiología y la de Clínica. En el año de 1789 se agregó a estas cuatro materias básicas la cátedra de Botánica, misma que ya cursaban los estudiantes de Farmacia.

Según señala Velasco Cevallos ${ }^{6}$, en su libro, Montaner trató de agregar las cátedras de Patología y Terapéutica, pero no tuvo éxito en esta propuesta, por lo que estas materias no se incluyeron en el programa académico.

Diez años después de su llegada a México, Andrés Montaner y Virgili sufrió una parálisis que le obligó a jubilarse en 1778. Montaner, ya jubilado, permaneció durante varios años más en la Ciudad de México, y no obstante su limitación física, continuó ejerciendo su profesión de Cirujano dentro del Colegio de San Ignacio de Loyola o de las Vizcaínas. En el año de 1779 , debido a la jubilación de Montaner, Manuel
Antonio Moreno Rodríguez fue designado Director del Colegio y Cirujano Mayor del Hospital Real de Naturales, por la Real Audiencia de México. Esto provocó una disputa entre Montaner y Moreno, ya que el primero se negó a dejar el cargo e incluso solicitó se le cancelara la jubilación; el virrey Martín de Mayorga apoyo a Moreno y le dio posesión de su cargo el 17 de marzo de $1779^{6}$.

Moreno ocupó un lugar destacado dentro de la sociedad novohispana, y además de su cargo en el Real Colegio de Cirugía también se desempeñó como Cirujano Segundo en el Hospital de San Andrés, hacia el año de $1782^{5}$.

En ese mismo año, el Tribunal del Protomedicato invitó a Andrés Montaner a formar parte de un grupo de cirujanos que evaluarían, en el Hospital de Naturales y en el Hospital de San Andrés, la aplicación de un nuevo tratamiento para la lepra a base de carne de lagartija. De los ocho enfermos que se atendieron, Montaner concluyó que ninguno de los casos había sanado ${ }^{6}$.

En el año de 1784, Montaner regresó a España, a Cataluña, donde continuó percibiendo su jubilación; después de este hecho, se pierde el rastro de este cirujano que años atrás había emprendido la encomienda de acudir a México para profesionalizar la enseñanza de la Cirugía en la Nueva España6.

Por su parte, Moreno continúo con una carrera ascendente, y en el año de 1790 presentó su obra Obstrucciones inflamatorias del hígado, la cual fue premiada por el Protomedicato. Moreno falleció en 1803, pero al parecer su situación económica no era muy buena y dejó en apremios económicos a su familia, de tal suerte que el cirujano Antonio Serrano, quien fungiría más tarde como Director del Real Colegio, cedió la mitad de su sueldo a la viuda y las hijas de Moreno ${ }^{5}$.

\section{Los últimos años}

Ya bajo la dirección de Antonio Serrano, el Real Colegio de Cirugía trató de conseguir su separación del Tribunal del Protomedicato. Serrano argumentaba que el Protomedicato no estaba capacitado para examinar a un cirujano, ya que ese tribunal únicamente estaba conformado por médicos. Esta separación permitiría al Real Colegio de Cirugía de México dar una mejor preparación a los cirujanos. Sin embargo, el Protomedicato defendió su derecho de mantener el control de la vigilancia tanto de las áreas médicas 
como de las quirúrgicas, y mantuvo su hegemonía hasta su desaparición en el año 1831.

Durante la Guerra de Independencia en la Nueva España, los recursos económicos tanto del Hospital Real de Naturales como del Real Colegio de Cirugía se vieron seriamente afectados. Durante las tres primeras décadas del siglo xix, el Colegio lucho por sostenerse a pesar de las circunstancias adversas. En agosto de 1818, Serrano solicitó al virrey Juan Ruiz de Apodaca el apoyo del erario real para sufragar los gastos del Colegio, o bien que se le permitiera cobrar a los estudiantes cuotas de inscripción y el pago de mensualidades para poder cubrir dichos gastos. De tal forma, el virrey tuvo que autorizar el cobro a los estudiantes del Colegio a partir del año $1820^{7}$.

Al consumarse la independencia, el Colegio tomó el nombre de Escuela Nacional de Cirugía, que durante el Imperio de Iturbide se conoció como Escuela Imperial de Cirugía, pero la situación económica continuaba siendo precaria, lo cual se agravó aún más con el cierre del Hospital Real de Naturales en 1822. No obstante, las clases de disección continuaron impartiéndose en dichas instalaciones, en tanto que la parte clínica se impartía en el Hospital de San Andrés.

En marzo de 1823, en respuesta a la convocatoria que hiciera Iturbide, el cirujano y diputado José Miguel Muñoz propuso ante la Comisión de Instrucción Pública que se unificaran la enseñanza de la medicina y la cirugía, y que se creara una Junta de Salud Pública que sustituyera al Protomedicato. Este último organismo, utilizando su influencia, evitó la aprobación de la propuesta ${ }^{8}$.

En 1827 hubo una gran carencia de fondos en la Escuela Nacional de Cirugía y no solo dejó de pagar su sueldo a los catedráticos, sino que los estudiantes tuvieron que prorratear los gastos para las composturas del edificio. En el año de 1830 aparecieron en el presupuesto del Gobierno Federal algunas partidas para sueldos y gastos de la Escuela, quedando esta desde entonces a expensas de la Tesorería General de la Nación. Las contribuciones de los estudiantes fluctuaban entre 257 y 171 pesos anuales ${ }^{11}$.

Ya para el año de 1833, las instalaciones de la Escuela Nacional de Cirugía se encontraban en gran deterioro, por lo que el director José Miguel García Cabezón solicitó al gobierno que se le asignaran nuevas instalaciones, lo cual no fue concedido. En diciembre de 1837 fallece José Miguel García Cabezón y el presidente Anastasio Bustamante nombra a Manuel Andrade y Pastor como Director de la
Escuela Nacional de Cirugía. Andrade era un cirujano latino, es decir, había estudiado tanto Medicina en la Universidad como Cirugía en la Escuela Nacional de Cirugía, y quizás esto influyó para que la Escuela Nacional de Cirugía, en el año de 1838, se incorporara al Establecimiento de Ciencias Médicas, finalizando de esta manera la trayectoria de la Escuela Nacional de Cirugía que había sido la continuación de aquel Real Colegio de Cirugía de Nueva España de la Ciudad de México, establecido por decreto real en $1768^{11}$.

En el Archivo Histórico de la Facultad de Medicina se encuentra el Libro de Matrículas del Real colegio de Cirugía de la Nueva España, el cual consigna las inscripciones de todos los alumnos que acudieron a él desde el inicio de sus actividades en 1770 hasta el año de 1834, un poco después de la reforma médica que reunió la enseñanza de la cirugía y la medicina en nuestro país. Este libro fue agregado al Archivo Histórico en el año de 2010, gracias al esfuerzo realizado por el Académico Dr. Enrique Graue Wiechers ${ }^{12}$.

\section{Conclusiones}

El Real Colegio de Cirugía de Nueva España, de la Ciudad de México, logró profesionalizar e institucionalizar la enseñanza de la Cirugía combinando la práctica con la teoría, y estableciendo programas de estudio con los conocimientos más avanzados para esa época en los campos de la anatomía, la fisiología y la técnica quirúrgica.

Montaner y Moreno trajeron la información más reciente del siglo xviii en estas áreas del conocimiento hasta el continente americano y dieron un impulso relevante a la práctica de la cirugía.

Fue el Colegio de Cirugía, sin duda, una institución que permitió una mayor capacitación de los cirujanos para poder dar una mejor atención a sus pacientes, dejando una enorme huella en la historia de la Medicina y la Cirugía de México.

\section{Financiación}

No se recibió patrocinio de ningún tipo para llevar a cabo este artículo.

\section{Conflicto de intereses}

Los autores declaran no tener ningún conflicto de intereses. 


\section{Responsabilidades éticas}

Protección de personas y animales. Los autores declaran que para esta investigación no se han realizado experimentos en seres humanos ni en animales.

Confidencialidad de los datos. Los autores declaran que en este artículo no aparecen datos de pacientes.

Derecho a la privacidad y consentimiento informado. Los autores declaran que en este artículo no aparecen datos de pacientes.

\section{Bibliografía}

1. Ocaranza F. El Real Colegio de Cirugía. En: Historia de la Medicina en México. México: Midy; 1934. p. 131-3.

2. Cronología de la estadística en México (1521-2008). Instituto Nacional de Estadística y Geografía (INEGI), Colección Memoria. México: INEGI; 2010. p. 10.
3. Ramírez-Ortega V. La enseñanza de las profesiones médica y quirúrgica hacia el final del régimen colonial. Rev Med Inst Mex Seguro Soc. 2010;48:159-62.

4. Cárdenas de la Peña E. Época colonial. En: Historia de la Medicina en la Ciudad de México. Colección Metropolitana. México: Talleres Gráficos de la Nación; 1976. p. 97-9.

5. Ramírez-Ortega V. El Real Colegio de Cirugía de Nueva España 17681833. La profesionalización e institucionalización de la enseñanza de la cirugía. Serie Los Cirujanos en la Nueva España IX. México: Instituto de Investigaciones Sociales. Universidad Nacional Autónoma de México; 2010. p. 105-79.

6. Velasco-Cevallos R. La cirugía mexicana en el siglo XVIII. México: Archivo Histórico de la Secretaría de Salubridad y Asistencia; 1946. p. 80-140.

7. Campos R, Ruiz-Llanos A. Adecuaciones interculturales en los hospitales para indios en la Nueva España. Gac Med Mex. 2001;137:595-608.

8. Morales-Cosme A, Aceves P, Gómez-Álvarez C, González-González E. Los cirujanos-médicos en México, 1802-1838. Llull: Revista de la Sociedad Española de Historia de las Ciencias y de las Técnicas. 2006;29:95-120.

9. Aguilar GF. Cirujanos de ayer. México: Bayer; 1938. p. 3-6.

10. Romero-Huesca A, Ramírez-Bollas J. La atención médica en el Hospital Real de Naturales. Cir Cir. 2003;71:496-503.

11. Valle RH. La cirugía mexicana en el siglo XIX. México: Tipografía Sag; 1942. p. 280-3.

12. Martínez-Barbosa MX. Los registros del Real Colegio de Cirugía. Rev Fac Med UNAM. 2015;58:32-42. 